\title{
Los ensayos clínicos y su contribución a la salud pública cubana
}

\author{
Clinical trials and their contribution to Cuban public health
}

\author{
Dr. C. Martha María Fors López \\ Centro Nacional Coordinador de Ensayos Clínicos (CENCEC). La Habana, Cuba.
}

\begin{abstract}
RESUMEN
La salud pública ha avanzado en los últimos años, en aras de mejorar la calidad de las evidencias científicas que sustentan las intervenciones y determinadas acciones de salud. La evaluación de la efectividad de las intervenciones en el ámbito clínico se basa en el diseño de ensayos clínicos controlados y aleatorizados, que sirven como una de las fuentes más confiables para la toma de decisiones en salud pública, a pesar de sus limitaciones. Se generan tensiones entre los investigadores e instituciones y los sistemas de salud pública, puesto que en ocasiones existen dudas de que los resultados de dichos estudios puedan generalizarse en la práctica. Las diferentes características que conforman el mundo de los investigadores y el de los responsables políticos, incluidos plazos, intereses y prioridades; pueden contribuir a estas tensiones e impedir la conexión entre los resultados de la investigación con los decisores en el campo de la salud pública. En este trabajo se hacen algunas consideraciones al respecto y se explica brevemente la situación en Cuba.
\end{abstract}

Palabras clave: ensayos clínicos aleatorizados, evidencias científicas, toma de decisión, salud pública.

\section{ABSTRACT}

Public health has advanced in recent years in order to improve the quality of scientific evidence supporting interventions and specific health actions. The evaluation of the effectiveness of interventions in the clinical setting is mainly based on the design of randomized controlled trials which serve, despite their limitations, as one of the most trusted sources for making public health decisions. On the other hand, there are 
controversies between the investigators, institutions and the public health systems, due to some uncertainty about the generalization of these results in the public health. The differences between the characteristics of the researchers' world and those of the world of policymakers, including deadlines, interests and priorities, can contribute to these controversies, thus preventing the connection of research findings to decisionmakers in this field. This paper made some considerations in this regard and briefly explained the present situation in Cuba.

Key words: randomized clinical trials, scientific evidence, decision making, public health.

\section{NTRODUCCI ÓN}

El concepto de salud pública se ha definido desde múltiples y variadas perspectivas. Entre las definiciones propuestas se encuentra la clásica de Acheson, ${ }^{1}$ que describe la salud pública como "la ciencia y el arte de prevenir la enfermedad, prolongar la vida y promover la salud mediante los esfuerzos organizados de la sociedad."

En los últimos años se ha introducido un término conocido como "la salud pública basada en la evidencia" entendida por J enicek, ${ }^{2}$ como "el uso consciente, explícito y juicioso de la mejor evidencia en la toma de decisiones sobre la atención a comunidades y poblaciones en el campo de la protección de la salud, la prevención de la enfermedad y el mantenimiento y mejora de la salud". Para la aplicación de esta definición se exige una sólida base tanto de conocimientos sobre la frecuencia, determinantes y consecuencias de una enfermedad, como de la seguridad, eficacia y efectividad de las diferentes alternativas para su tratamiento, evaluadas fundamentalmente a través de ensayos clínicos controlados y aleatorizados (ECA).

Estos estudios son esenciales para evaluar la eficacia, la efectividad y la seguridad y la calidad de nuevas intervenciones terapéuticas donde los resultados pueden extrapolarse de forma segura, según la mayoría de los expertos, a otras poblaciones. Los ECA permiten obtener el nivel de evidencia más alto para demostrar que el procedimiento médico que se realiza es el más adecuado con los conocimientos científicos que existen en ese momento, debido al diseño del estudio, donde las variables estadísticas están controladas para evitar posibles sesgos.

Sin embargo, en el caso de las evaluaciones de intervenciones de salud pública, los resultados de los ECA están sujetos a modificación de su efecto en las diferentes poblaciones por lo que en estos casos se valoran de forma especial la validez interna y la validez externa de estos resultados.

El éxito de algunas corrientes como la Medicina basada en la evidencia (MBE) y de sus herramientas así como el trabajo de algunas organizaciones como la Colaboración Cochrane, han alentado la extensión de los diseños de ECA a los campos de la salud pública y a las políticas de salud. Los ensayos clínicos pueden contribuir a uniformar la práctica médica, elevar la calidad de la atención al paciente y mejorar la organización de un servicio de salud, por lo que tienen el potencial de salvar vidas, de reportar beneficios en la mejora de la calidad de vida de los pacientes, de contribuir a cambios 
en patrones de manejo de enfermedades así como de modificar en ocasiones indicadores importantes de salud.

Por estas razones, son necesarios pasos formales para extender los resultados y utilizar la evidencia científica obtenida en estos estudios de forma tal que puedan ser incorporados lo antes posible en las políticas de salud pública y por tanto facilitar la toma de decisiones en este campo.

La aplicación de los resultados de la investigación clínica a la práctica médica, incluye no solo el mejor uso de los medicamentos evaluados y de las intervenciones conductuales y organizacionales de los servicios de salud sino además el conocimiento sobre la utilización de numerosas de tecnologías sanitarias. Estos resultados brindan elementos que facilitan la toma de decisiones clínicas basadas en la mejor evidencia disponible.

Mientras que la ciencia básica, la clínica y la investigación en salud contribuyen a la obtención de resultados necesarios para mejorar la salud de la sociedad, la investigación en salud pública permite la comprensión de la distribución de la salud y la enfermedad en la población, los factores determinantes de esta distribución y las formas en que el sistema de salud y las políticas de salud podrían reducir la carga de una determinada enfermedad.

La relación entre los ensayos clínicos y la salud pública genera tensiones, pues la ejecución de este tipo de estudio se realiza bajo condiciones experimentales y para un determinado grupo de pacientes delimitados por criterios de inclusión generalmente estrictos, sin embargo, existe necesidad de que estos estudios sean relevantes para toda la comunidad y no para parte de la misma. Esta razón sirve como base para distinguir los ECA: entre ensayos pragmáticos y explicativos, ${ }^{3}$ entre los ensayos explicativos y la gestión, ${ }^{4}$ o entre los ensayos mecánicos y prácticos. ${ }^{5}$ El enfoque explicativo enfatiza en la obtención de nuevos conocimientos e información y trata de estimar la efectividad del método empleado en el estudio mientras que el enfoque pragmático se centra en la toma de decisiones y trata de estimar la efectividad de uso.

Los ensayos deben diseñarse además para cubrir lagunas en el conocimiento científico, pero el logro de este objetivo depende de un acuerdo entre las partes interesadas acerca de la interpretación del estado de la evidencia actual y las prioridades para la investigación. Se necesita un balance entre las prioridades de los promotores de la investigación, los cuales asignan recursos para estos estudios y el estado del conocimiento científico y los hechos científicos existentes sobre la enfermedad en cuestión, los tratamientos evaluados por diferentes estudios y si realmente existe un problema de salud a resolver.

En Cuba, el Centro Nacional Coordinador de Ensayos Clínicos (CENCEC), institución subordinada la Ministerio de Salud Pública de Cuba, y que sirve a sus intereses tiene como uno de sus objetivos asegurar la evaluación clínica de productos médico farmacéuticos, y biotecnológicos así como de equipos médicos cubanos de acuerdo a estándares internacionales. Esta institución es líder en este campo dentro del país y tiene 20 años de experiencia en el diseño y conducción de ensayos clínicos de todas las fases de desarrollo de un nuevo fármaco. Posee una alta especialización en el área de ensayos clínicos para evaluar nuevas terapias para el tratamiento de enfermedades neoplásicas y también ha trabajado en la investigación de nuevos tratamientos para otras indicaciones médicas. El objetivo de este trabajo es abordar la interrelación entre los ensayos clínicos y los sistemas de salud pública y el papel de los ECA como una de las fuentes de obtención de resultados científicos para la toma 
de decisiones por las autoridades de la salud pública. Se describe brevemente el papel del CENCEC dentro del Sistema Nacional de Salud de Cuba.

\section{¿QUÉ TI PO DE TENSI ONES PUDI ERAN GENERARSE ENTRE LA I NVESTI GACIÓN CLÍ NI CA Y LA SALUD PÚBLI CA?}

En la actualidad, alrededor de unos 50000 ensayos clínicos se ejecutan a nivel mundial. Más del $40 \%$ de estos nuevos estudios se están realizando en áreas geográficas donde habitualmente no se hacía este tipo de investigación, países que están experimentando un cambio en el cuadro epidemiológico, asociado con una disminución de los recursos de salud disponibles, y que tienen poca participación en el mercado farmacéutico mundial.

Los temas mencionados anteriormente están estrechamente conectados a las consideraciones de equidad y justicia.

Los estudios que involucran sujetos humanos se desarrollan siguiendo documentos de alcance nacional e internacional como regulaciones, normativas y leyes que tienen como objetivo proteger al individuo bajo estudios experimentales y que contemplan principios universales los cuales no deben ser violados. Por ejemplo, la evaluación del perfil de riesgo beneficio de un determinado tratamiento bajo ensayo clínico, es una preocupación de todos los implicados ya que el bienestar de los participantes en sí es de importancia ética moral fundamental. Esto exige que los beneficios que obtendrán los sujetos bajo investigación tengan que ser superiores a cualquier riesgo que pueda ocurrir durante el estudio. Estos aspectos también son importantes para asegurar que los centros de investigación reflejen los términos justos de la cooperación social en este sentido.

La falta de adecuación a las necesidades de salud de la comunidad puede afectar a la evaluación del riesgo beneficio de un nuevo tratamiento. En parte, a medida que decrece el valor social de los ensayos clínicos, se hace más difícil justificar algunos riesgos a que son sometidos los participantes. Generalmente los pacientes no son compensados monetariamente por participar en los ensayos clínicos, simplemente esperan tener la posibilidad de obtener un beneficio directo derivado del estudio o la posibilidad de que otros sujetos puedan beneficiarse a largo plazo de los medicamentos evaluados. Cuando la investigación es relevante para satisfacer las necesidades de salud de la comunidad, la participación de los sujetos es esencial como apoyo al sistema de salud pública y se considera, además, como una forma de contribuir a un bien público importante y al desarrollo de la industria farmacéutica y biotecnológica, como ocurre en Cuba.

Cuando existen importantes diferencia de opiniones entre los patrocinadores de la investigación y el sistema de salud pública, los ensayos clínicos no tendrían razón de ser, pero la realidad es muy diferente. Todo estudio de este tipo debe estar claramente vinculado a los objetivos de salud o de desarrollo vislumbrados en los programas aprobados por el sistema de salud pública, sin embargo, en muchos de los países esto no se garantiza.

Los ensayos clínicos pueden tener un impacto directo sobre la distribución de oportunidades en una comunidad determinada y contribuir a mitigar algunas desigualdades sociales que perjudican a determinadas poblaciones vulnerables. Se debe asegurar por todos los medios posibles que la investigación no genere o exacerbe las desigualdades inaceptables en la comunidad donde se realiza el estudio clínico. 
Actualmente los ensayos clínicos se están desarrollando en áreas geográficas no habituales y se integran de forma compleja a la salud pública y a la calidad de la atención médica en ese contexto. El proceso de desarrollo de nuevos medicamentos ha sido marcado por el sello de una serie estructurada de prácticas inherentes a la industria farmacéutica, como se ha desarrollado en América del Norte y en algunos países europeos desarrollados. Ejemplos de estas prácticas han sido la utilización de minorías vulnerables y los llamados pacientes "cooperativos" y los "conejillos de indias" profesionales, y el poder que ejerce la industria farmacéutica sobre las decisiones y la política de reglamentación farmacéutica en estos países. ${ }^{6-8}$

Para la investigación en salud no siempre se pueden ejecutar diseños clásicos aleatorizados y controlados de forma estricta, a veces por criterios éticos, otras por razones logísticas y otras, por imposibilidad conceptual, ${ }^{9-11}$ de ahí que las intervenciones en la mayoría de ocasiones son de tipo multicomponente, con lo cual la interpretación de resultados es compleja. ${ }^{12}$

La evaluación de la efectividad de las intervenciones en salud pública no debe basarse exclusivamente en el ensayo clínico, pues este además de presentar algunas limitaciones de índole práctico, podría resultar inadecuado: en ocasiones limitada validez externa (considera muestras de sujetos, ámbitos e intervenciones poco generalizables), desvincula la intervención de su contexto habitual de aplicación (y elimina, por tanto, la probable influencia del contexto en los resultados de la intervención) y no tiene en cuenta el proceso de implementación de la intervención.

La importancia de la investigación clínica para el bienestar de un gran número de personas ha aumentado en los últimos años pero los beneficios derivados de la investigación globalizada se distribuyen de manera desigual. Las ideas institucionales actuales sobre la protección del paciente siguen siendo bastante restrictivas, y la dimensión de los daños que pueden ocurrir en la etapa de experimentación podría ser considerable.

La posibilidad de elegir por ejemplo pacientes vírgenes de tratamiento permite la obtención de resultados eficientes, libres de "ruido estadístico", pero se crean serias dudas acerca de la generalización de los datos.

Por ejemplo ¿cómo estos resultados cada vez son más generalizables a la población que se nutre de mercados saturados de medicamentos? En la práctica médica habitual es difícil hallar a pacientes con enfermedades crónicas que no tomen más de un medicamento, sin embargo, generalmente los criterios de inclusión de sujetos en un estudio, limitan la entrada de aquellos pacientes que estén tomando medicamentos para el control de su enfermedad.

¿Cómo es posible que aún entren al mercado farmacéutico medicamentos ineficaces y con perfiles de seguridad con problemas? Los estudios clínicos generalmente tienen tamaños de muestra pequeños y se ejecutan siempre bajo condiciones experimentales controladas, una vez registrado el nuevo medicamento por las agencias reguladoras comienza el proceso de su comercialización y por tanto su extensión a gran número de sujetos. Una vez que el medicamento es utilizado por un número elevado de personas pudieran aparecer eventos adversos a largo plazo y problemas de efectividad de este medicamento lo que en ocasiones conlleva a su retirada del mercado.

Es por razones como estas que los sistemas de salud pública deben ocuparse de que estas situaciones no ocurran. Estas prácticas se permiten en ausencia de instituciones gubernamentales capaces de controlar la entrada de medicamentos ineficaces en el mercado y de monitorear sus efectos secundarios a largo plazo, por no mencionar 
que, en muchos países, los pacientes aún carecen de recursos legales para compensar los daños ocasionados durante y después de la participación en un ensayo clínico.

\section{¿LOS ENSAYOS CLí NI COS CONTROLADOS, PI LAR DE LAS EVI DENCIAS CI ENTÍ FI CAS?}

Los avances en distintas ramas científicas en el siglo xx favorecen el surgimiento de un nuevo paradigma relacionado con la práctica médica. Este paradigma se basa en evidencias científicas y permite ofrecer servicios médicos de mayor calidad con costos asociados menores. Estimula además, la introducción sistemática del conocimiento científico a la práctica profesional, la educación continua y el desarrollo de la investigación clínica.

La práctica médica llamada también práctica clínica, puede considerarse como el proceso de interacción médico paciente. La variabilidad en la práctica puede ocurrir debido a incertidumbres, ignorancia y existencia de recursos limitados que originan diferencias en la toma de decisiones sobre pacientes individuales. La hipótesis más extendida actualmente sobre el origen de estas diferencias es la que relaciona la variabilidad con el grado de incertidumbre que acompaña a la toma de decisión, por lo que es necesario que los decisores de políticas de salud se preocupen por generar y publicar recomendaciones para estas circunstancias.

El ECA es considerado el pilar metodológico más importante sobre el que se sustenta la investigación clínica moderna, constituye un motor impulsor para la Medicina basada en evidencia y es uno de los primeros niveles de obtención de resultados científicos. ${ }^{13}$ La ingente cantidad de conocimiento publicado no se puede abordar sin herramientas que permitan el acceso a todas las fuentes posibles para recuperar y seleccionar los trabajos científicos más significativos. Ya no es válido, a pesar de que continúa desempeñando un papel muy importante, confiar en la última edición del tratado de referencia que actualiza la forma de abordar, diagnosticar o tratar una enfermedad concreta. El conocimiento cambia y se modifica rápidamente, y hay que estar alertas para actuar, o procurar actuar, en todo momento con el mejor y más efectivo conocimiento científico. Es preciso que todo este conocimiento responda al más alto grado de rigor científico (experimentación y reproducibilidad) y se distancie de lo que es simplemente anecdótico o espurio. ${ }^{14}$ Así aparecen diferentes instrumentos como las Guías de Practica Clínica, que utilizan la mejor evidencia disponible y utilizan en su elaboración métodos como las revisiones sistemáticas y los metanálisis de ensayos clínicos controlados.

El grado de conocimiento existente y la profundidad que este ha adquirido en diversas materias relacionadas con las investigaciones biomédicas resultan inimaginables, sin embargo, en ocasiones este conocimiento no se introduce en la práctica clínica ni en las políticas de salud con la rapidez con que deberían.

\section{¿Cómo repercuten los ensayos clínicos controlados y aleatorizados cubanos en el Sistema Nacional de Salud?}

El Sistema Nacional de Salud cubano tiene cualidades extraordinarias como son la accesibilidad y gratuidad de los servicios. Se caracteriza además por la introducción y extensión de los adelantos de la ciencia y la técnica y la presencia de la colaboración internacional en diversos campos. El Ministerio de Salud Pública como organismo rector tiene entre otras funciones: ejercer la evaluación, el registro, la regulación y el control de los medicamentos de producción nacional y de importación, equipos 
médicos y material gastable y otros de uso médico; regular y controlar la aprobación, ejecución y evaluación de las investigaciones biomédicas o de cualquier tipo que se realice directamente en seres humanos.

El desarrollo de nuevos fármacos es un proceso complejo y la evaluación clínica de estos compuestos es uno de sus componentes fundamentales. En Cuba, existe un desarrollo importante de la biotecnología y de centros de investigación que producen novedosos productos que necesitan de la evaluación clínica según estándares nacionales e internacionales.

Por la necesidad imperiosa de lograr buena calidad y velocidad en la obtención de resultados de las investigaciones clínicas que evalúan productos de la industria biotecnológica cubana, elementos que permiten su registro sanitario tanto en Cuba como en los países donde se pueden comercializar, se crea a fines de 1991, una organización para el diseño y conducción de los ensayos clínicos concebida como Centro Nacional y de forma inmediata, como red de coordinación (CENCEC) ${ }^{15}$

La investigación clínica se realiza en unidades asistenciales que forman parte del Sistema Nacional de Salud del país y que deben cumplir ciertos requisitos para garantizar la buena calidad de estas investigaciones. Los sitios clínicos involucrados en la ejecución de los ensayos clínicos deben contar con personal capacitado en las normas de Buenas Prácticas Clínicas (BPC), las cuales son guías internacionales para el desarrollo de investigaciones en sujetos humanos que garantizan la confiabilidad de los resultados obtenidos. En Cuba, el Centro para el Control Estatal de Medicamentos, Equipos y Dispositivos Médicos (CECMED), posee normas de BPC adaptada a las condiciones del país pero que se adhieren a las internacionales, y se encarga, además, de promover y proteger la salud pública a través de un sistema regulador que garantiza el acceso oportuno al mercado de productos con calidad, seguridad, eficacia e información veraz para su uso racional; más información disponible en su sitio. ${ }^{16}$

La investigación que se desarrolla en el Sistema Nacional de Salud, debe responder a las prioridades y metas de las Áreas Estratégicas de las Proyecciones de la Salud Pública y todos los proyectos de investigación deben ser aprobados por los consejos científicos y los comités de ética de investigación (CEI) de las instancias correspondientes. En el caso de los ECA es requisito indispensable tener una aprobación del CEI para comenzar el estudio en cada sitio, cuya función primordial es proteger a los sujetos que se incluyen en la investigación y eliminar la utilización de sujetos como los conocidos "conejillos de india", práctica frecuente en algunos países de la región.

En el caso particular de Cuba, existe una integración entre los diferentes implicados en la realización de los ensayos clínicos (MINSAP, CITMA, MINBAS, entre otros) y responsabilidad compartida en la oportuna publicación de los resultados de las investigaciones, la comunicación al público y a los profesionales de la salud, según corresponda. Por otra parte, la responsabilidad en la aplicación de los resultados a las políticas de salud pública del país recae en las autoridades de salud, sin embargo, existe una conciliación de los intereses de los implicados para lograr eliminar posibles diferencias entre los patrocinadores de las investigaciones y los intereses públicos de salud. El Sistema Nacional de Salud cubano y los promotores de ensayos clínicos, además, garantizan los recursos materiales que permiten a los sitios clínicos cumplir con lo establecido en los protocolos de investigación.

En Cuba se ha otorgado siempre gran importancia a los ensayos clínicos dada fundamentalmente, por su contribución a la educación de los médicos en favorecer el sentido crítico y la correcta evaluación del uso de nuevos medicamentos y otras 
terapias médicas así como de medios de diagnóstico, lo que contribuye a la elevación de la calidad de los servicios médicos.

EI CENCEC ha conducido 127 ECA hasta el 2011, no solo para evaluar nuevos productos para registro sanitario, sino además para facilitar su extensión a toda la población a través de la implementación de estudios fase IV. Los estudios de poscomercialización de seguridad y confirmación de efectividad de productos ya registrados han permitido la implementación de algunos programas nacionales a través del Ministerio de Salud Pública. Se han diseñado además ensayos clínicos específicamente en interés de la salud pública, con el objetivo de solucionar problemas serios de salud o epidemias. ${ }^{15}$

Además de los resultados de los estudios que apoyan el registro sanitario de los nuevos tratamientos, los ensayos clínicos tienen otros beneficios claros, entre ellos, la mejoría que se produce en los indicadores de salud que se modifican por los resultados de la introducción de un nuevo producto o de una nueva indicación, así como los cambios que se producen en los patrones de atención médica de la enfermedad en que se evalúa el producto, ya que se exige para la aceptación del protocolo del ensayo clínico, los mejores estándares de diagnóstico, evaluación y tratamiento de la enfermedad.

En ocasiones, el ensayo clínico contribuye a la introducción de nuevos métodos para el diagnóstico, nuevas tecnologías de evaluación y nuevas terapéuticas asociadas al producto en estudio como los tratamientos concomitantes o tratamientos activos utilizados en los grupos controles. Otros beneficios incluyen la formación y perfeccionamiento de los recursos humanos que participan en el estudio, en la propia especialidad, ya que por el propio ensayo clínico, se ven obligados a estudiar aspectos del estado del arte de la enfermedad o entidad en evaluación y en otros campos de la investigación, como son las BPC, aspectos éticos y los principios de la MBE que contribuye a un mayor rigor científico y todo esto por ende, una mejor atención a los pacientes.

A través de los ensayos clínicos puede lograrse una mejor organización y coordinación entre servicios médicos dentro de una institución además del aseguramiento de insumos médicos que se requieren de forma estable y preferiblemente certificados para los laboratorios, servicios y farmacias.

El proceso del monitoreo de los ensayos clínicos aleatorizados y controlados en el país, permite conocer las desviaciones del protocolo y el incumplimiento de las BPC pero además detecta y reporta, las desviaciones de los patrones de atención que no permiten la inclusión o la evaluación adecuada de los pacientes y que se relacionan directamente con la práctica médica. También puede lograrse un incremento de la calidad de las historias clínicas, y que, según las necesidades reguladoras, pueden ser auditadas o inspeccionadas por autoridades nacionales y extranjeras.

\section{CONSI DERACI ONES FI NALES}

Las investigaciones clínicas en el contexto cubano se abordan teniendo en cuenta los problemas de salud identificados en la población y se desarrollan con alto rigor científico lo que permite obtener resultados que pueden contribuir a la toma de decisiones en el ámbito de la salud pública. 
El ensayo clínico controlado a pesar de algunas limitaciones representa un papel importante en la obtención de las evidencias científicas necesarias para sustentar estas decisiones ya que constituye el patrón de oro de evaluación de nuevos fármacos y propulsor de la Medicina basada en la evidencia.

Los ensayos clínicos diseñados según los intereses de los sistemas de salud pública y de la población blanco a la cual van dirigidas las nuevas terapias evaluadas, constituyen un camino imprescindible para que la salud pública eleve su nivel de organización y de eficiencia, lo que permite a su vez generar investigaciones científicas y obtener resultados sólidos para tomar las decisiones adecuadas en beneficio de la humanidad.

\section{Agradecimientos}

Mis agradecimientos a la Dra. María Amparo Pascual López, Directora del Centro Nacional Coordinador de Ensayos clínicos (CENCEC) y a la Dra. Maytee Robaina García por su contribución en la redacción de este trabajo.

\section{REFERENCI AS BI BLI OGRÁFI CAS}

1. Acheson D. Report of the Committee of Inquiry into the future development of the Public Health functions and Community Medicine. London: HMSO; 1988:

2. Jenicek M. Epidemiology, evidenced-based medicine, and evidence-based public health. J Epidemiol. 1997; 7: 187-97.

3. Schwartz D, Lellouch J. Explanatory and pragmatic attitudes in therapeutical trials. J Chronic Dis. 1967;20:63748.

4. Sackett DL.The principles behind the tactics of performing therapeutic trials. How to Do Clinical Practice Research. 3rd edition. Lippincott: Williams and Wilkins; 2006.

5. Karanicolas PJ, Montori VM, Devereaux PJ, Schunemann H, Guyatt GH. A new "mechanistic-practical" framework for designing and interpreting randomized trials. J Clin Epidemiol. 2009;62(5):479-84.

6. Goozner M. The $\$ 800$ million pill: The truth behind the cost of new drugs. Berkeley: University of California Press; 2004.

7. Kassirer JP. On the take: How medicine's complicity with big business can endanger your health. Oxford: Oxford University Press; 2004.

8. Moynihan R, Cassels A. Selling sickness: How the world's biggest pharmaceutical companies are turning us all into patients. New York: Nation Books; 2005.

9. Thomson H, Hoskins R, Petticrew M, Ogilvie D, Craig N, Ouinn T, et al. Evaluating the health effects of social interventions. BMJ . 2004;328:282-5.

10. Victora CG, Habicht JP, Bryce J. Evidence-based public health: moving beyond randomized trials. Am J Public Health. 2004; 94:400-5.

http://scielo.sld.cu 
11. Vera M. Evaluar intervenciones sanitarias sin experimentos. Gac Sanit. $2003 ; 17(3): 238-48$

12. Campbell M, Fitzpatrick R, Haines A, Kinmonth A, Sandercock P, Spiegelhalter D, et al. Framework for design and evaluation of complex interventions to improve health. BMJ . 2000; 321:694-6.

13. Devereaux PJ, Manns BJ, Ghali WA, Quan H, Guyatt GH. The reporting of methodological factors in randomized controlled trials and the association with a journal policy to promote adherence to the Consolidated Standards of Reporting Trials (CONSORT) checklist. Control Clin Trials. 2002;23:380-8.

14. Pons JMV. Gestión clínica basada en las fuentes de información: las guías de práctica clínica. Una nueva forma de trabajar que mejora las decisiones. Programa, ponencias, experiencias y comunicaciones. Barcelona: Congreso Gestión Clínica; 2003.

15. Pascual MA, Jiménez G, Torres A, Fors M, López I. Surgimiento, evolución y principales resultados del Centro Nacional Coordinador de Ensayos Clínicos. Rev Cubana Farm [Internet]. 2011 [citado 4 Ago 2012]; 45(1):4-18. Disponible en: http://scielo.sld.cu/pdf/far/v45n1/far02111.pdf

16. Centro para el Control Estatal de Medicamentos, Equipos y Dispositivos Médicos [Internet]. La Habana: el Centro; 1997-2012 [actualizado 24 Jul 2012; citado 4 Ago 2012]. Disponible en: http://www.cecmed.sld.cu/

Martha María Fors López. Unidad de Cooperación Internacional. Centro Nacional Coordinador de Ensayos clínicos (CENCEC). Calle 21 No. 19910 e/198 y 200, Atabey, Playa. La Habana, Cuba. Correos electrónicos: mfors@infomed.sld.cu; martaf@cencec.sld.cu 